\title{
New atomistic model of pyrrole with improved liquid state properties and structure
}

\author{
Marina Macchiagodena ${ }^{1}$ (1) | Giordano Mancini ${ }^{1,2}$ (1) | Marco Pagliai ${ }^{3}$ () । \\ Gianni Cardini ${ }^{3}$ (1) | Vincenzo Barone ${ }^{1,2}$ (1) \\ ${ }^{1}$ Scuola Normale Superiore, Piazza dei Cavalieri 7, I-56126 Pisa, Italy \\ ${ }^{2}$ Istituto Nazionale di Fisica Nucleare (INFN) sezione di Pisa, Largo Bruno Pontecorvo 3, I-56127 Pisa, Italy \\ ${ }^{3}$ Dipartimento di Chimica "Ugo Schiff", Università degli Studi di Firenze, Via della Lastruccia 3, I-50019 Sesto Fiorentino, Italy
}

\section{Correspondence}

Vincenzo Barone, Scuola Normale

Superiore, Piazza dei Cavalieri 7, I-56126

Pisa, Italy.

Email: vincenzo.barone@sns.it

\section{Funding information}

European Research Council under the European Union's Seventh Framework Programme, Grant/Award Number: FP/ 2007-2013/ERC Grant Agreement Number: 320951; Scuola Normale Superiore grant "Giovani Ricercatori 2015"

\begin{abstract}
Structural characterization of liquid pyrrole has been obtained by performing classical molecular dynamics simulations with a new parameterization of electrostatic interactions. Despite the relatively simple molecular structure of pyrrole, a correct and accurate representation of its intermolecular interactions in bulk phase is a challenging task, since these are affected at short range by the quadrupole-quadrupole term. This new parameterization permits not only to correctly describe the liquid structure but also to obtain macroscopic properties in excellent agreement with experiments.
\end{abstract}

\section{KEYWORDS}

bulk properties, force field, molecular dynamics, quadrupole moment

\section{1 | INTRODUCTION}

Pyrrole $\left(\mathrm{C}_{4} \mathrm{H}_{5} \mathrm{~N}\right)$ is one of the simplest and most representative heteroaromatic molecules. Its five-membered ring is the building block of several molecules of biological and pharmacological relevance, such as amino acids, indole alkaloids, ${ }^{[1]}$ and porphyrins. ${ }^{[2]}$ Some molecules containing the pyrrole moiety find application also as therapeutic compounds like fungicides, antibiotics, anti-inflammatory drugs, ${ }^{[3]}$ cholesterol reducing drugs, ${ }^{[4]}$ and antitumor agents. ${ }^{[5]}$ Pyrrole has been used in technological applications like, for example, polymers with remarkable electric conductivity ${ }^{[6]}$ and in dye-sensitized solar cells. ${ }^{[7,8]}$

The structure of pyrrole (see Figure 1) features an $\mathrm{N}-\mathrm{H}$ group and a $\pi$ system, which permit effective self-aggregation into clusters through the formation of a weak $\mathrm{N}-\mathrm{H} \cdots \pi$ hydrogen bonds. This behavior has been confirmed by experimental and theoretical studies which have shown the tendency to form T-shaped aggregates, ${ }^{[9-13]}$ which are typical structures found in the so called "quadrupolar crystals". In particular, it has been observed in microwave spectroscopy experiments ${ }^{[14]}$ that the angle formed by two monomers approaches to $\sim 55^{\circ}$ with a distance between the centers of mass of $\sim 4.1 \AA$.

The nature of the $\mathrm{N}-\mathrm{H} \cdots \pi$ hydrogen bond and the relevance of high multipole contributions to the liquid structure are not yet completely understood, especially in the liquid state. In fact, although several studies about small clusters are available, only few experimental and theoretical studies have been performed on liquid pyrrole. ${ }^{[13,15-17]}$ Hence, in this work, we present a computational study on liquid pyrrole with the purpose of accurately determining its structural features and bulk properties.

The structure of liquid pyrrole and its evolution over time has been described by classical molecular dynamics (MD), which allows to estimate macroscopic properties from microscopic models. To obtain results comparable with experiments, it is mandatory to employ an accurate force field (FF), which describes the interactions at atomic and molecular level using a set of functional forms based on a limited number of parameters. Some properties are very sensitive to the electrostatic part of the force field, which in the most widely used FFs is described using partial atomic charges. 


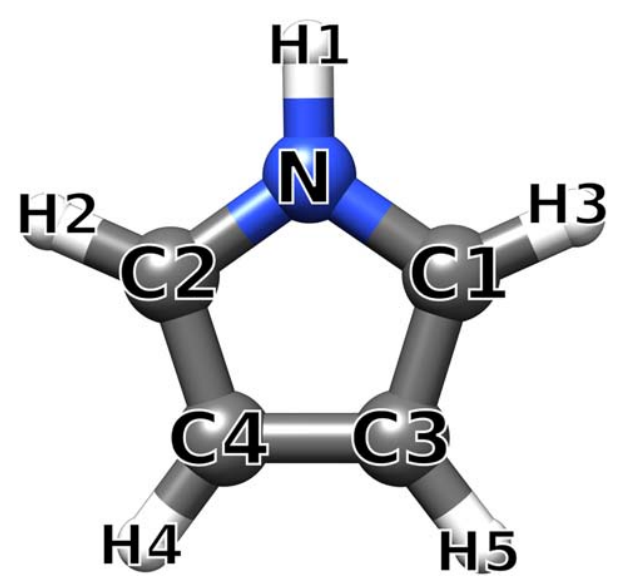

FIGURE 1 Pyrrole structure and atom labeling

General purpose FFs, trained to describe a large number of molecules in a given domain (e.g., OPLS ${ }^{[18,19]}$ for organic molecules) often fail to be very accurate in the reproduction of some macroscopic observables; this may be due to limits in the model but it is also unavoidable because of the need of transferability. For instance, estimations of the static dielectric constant with the common available FFs yields an average error of about $10 \%$. ${ }^{\text {[20] }}$ Several strategies have been employed to determine effective values for these quantities; one of the most adopted approaches relies in optimizing their values to reproduce the quantum mechanical (QM)-derived electrostatic potential of a molecule. ${ }^{[21]}$ In the present study, the electrostatic parameters have been obtained through Charge Model $5^{[22]}$ (CM5), performing the calculations at the B3LYP/6-31 + G(d) level of theory and taking into account bulk solvent effects by means of the conductor-like polarizable continuum model ${ }^{[23]}$ (C-PCM). The atomic charges have been further adjusted applying a fitting procedure to reproduce dipole and quadrupole moments determined by DFT calculations. For small rigid molecules, it is important to take into account the correct multipolar distribution to reproduce the nearest neighbor orientation. Furthermore, the T-shaped pair, characterizing the pyrrole structure, has been found one of the lowest energy configurations from the electrostatic interactions of quadrupole moments of the same sign. ${ }^{[2]}$ A variety of approaches have been attempted to describe the quadrupole moment in aromatic groups, including central multipoles, ${ }^{[25]}$ distributed multipoles, and off-center charges. ${ }^{[26,27]}$ Here, we want to highlight the possibility to describe correctly the pyrrole bulk properties and structure using simple point charges. In this respect, we have applied a fitting procedure to reproduce the dipole and quadrupole moment obtained from QM calculations.

\section{2 | METHODOLOGY}

\section{1 | Force field parameterization}

The pyrrole molecular structure has been optimized performing DFT calculations at the B3LYP/6-31 + G(d) level of theory with Gaussian 09 suite of programs, ${ }^{[28]}$ describing the solvent effects with the polarizable continuum model within the C-PCM formalism. ${ }^{[23]}$ Since the parameters to describe pyrrole as a solvent were not included in the adopted version of Gaussian package, tetrahydrofurane has been used as reference solvent due to the very similar dielectric constant. Using the same protocol adopted in the parameterization of the electrostatic part of formamide molecules, its $\mathrm{N}$-methyl derivatives ${ }^{[29]}$ and pyridine (nicotine), ${ }^{[30,31]}$ a CM5 population analysis ${ }^{[22]}$ has been carried out to obtain the initial atomic charges, as reported in Table 1. To improve the description of the molecular multipoles, the atomic charges have been adjusted through a fitting procedure, using as reference the dipole and traceless quadrupole moments obtained at the end of the optimization procedure, as reported in Table 1.

The intramolecular and intermolecular potential have been described by employing the OPLS/AA ${ }^{[18,19]}$ force field.

\section{2 | Molecular dynamics simulations}

Classical molecular dynamics simulations of pure pyrrole liquid solution were carried out using GROMACS v. 4.6 .5 software. ${ }^{[32]}$ The simulation was performed on a system containing 500 molecules and employed a cubic box with periodic boundary conditions. Initially a steepest descent energy

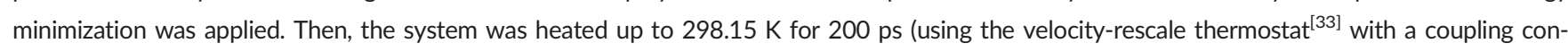
stant, $\tau$, of 0.1 ps). Starting from the last configuration obtained from the NVT equilibration, a simulation in the NPT ensemble (using the ParrinelloRahman barostat ${ }^{[34]}$ with $\tau=0.1 \mathrm{ps}$ ) was performed and the system was let to converge to uniform density. An additional simulation was run in the NVT ensemble using the average box dimensions obtained from the NPT simulation and increasing the integration time step from 0.2 to 2.0 fs, fixing the fastest degrees of freedom with the LINCS $^{[35]}$ algorithm. Finally, a sampling simulation was carried out for 50 ns with an integration time step $\delta \mathrm{t}=2.0 \mathrm{fs}$, without to run multiple replicas of the trajectory since local structure of a pyrrole first shell loses completely its memory in about 5 
TABLE 1 The CM5 charges (e) for pyrrole calculated at B3LYP/6-31 + G(d) level of theory taking into account the solvent (tetrahydrofurane) effect by means of C-PCM and used in the simulation (adjusted)

\begin{tabular}{|c|c|c|c|}
\hline \multirow[b]{2}{*}{ Atom } & \multicolumn{3}{|l|}{$Q$ (e) } \\
\hline & CM5 & Adjusted & $\Delta$ (CM5-Adj) \\
\hline N1 & -0.408403 & -0.382947 & -0.025456 \\
\hline $\mathrm{C} 1$ & -0.028826 & -0.038620 & 0.009794 \\
\hline $\mathrm{C} 2$ & -0.028826 & -0.038620 & 0.009794 \\
\hline $\mathrm{C} 3$ & -0.155496 & -0.161177 & 0.005681 \\
\hline $\mathrm{C} 4$ & -0.155496 & -0.161177 & 0.005681 \\
\hline $\mathrm{H} 1$ & 0.350463 & 0.371429 & -0.020966 \\
\hline $\mathrm{H} 3$ & 0.117192 & 0.119157 & -0.001965 \\
\hline $\mathrm{H} 2$ & 0.117192 & 0.119157 & -0.001965 \\
\hline H5 & 0.096100 & 0.086399 & 0.009701 \\
\hline $\mathrm{H} 4$ & 0.096100 & 0.086399 & 0.009701 \\
\hline
\end{tabular}

$\begin{array}{lr}\mu(z)(D) & 2.4930 \\ Q Q(x x)(D \cdot A ̊) & -6.5926 \\ Q(y y)(D \cdot A ̊) & 1.5068 \\ Q(z z)(D \cdot \AA) & 5.0858\end{array}$

In the column $\Delta$ (CM5-Adj) is reported the difference between the calculated and used charges. The dipole moment ( $\mu$ ) and quadrupole moment (Q) components obtained from QM calculations and used in the fitting procedure are also reported.

ps, as proved by the calculation of dimers lifetime. Electrostatic interactions were evaluated using the particle-mesh Ewald (PME) ${ }^{[36]}$ method with a grid spacing of $1.2 \AA$, a real-space cutoff of $1.2 \mathrm{~nm}$ and a spline interpolation of order 4 . Van der Waals interactions were computed applying a cutoff of $1.2 \mathrm{~nm}$. Long range dispersion corrections have been using for energy and pressure. Furthermore, since the dipole moment fluctuations are sensitive to the boundary conditions applied we have fixed the external dielectric constant to the experimental value.

\section{3 | Ab initio molecular dynamics simulations}

Ab initio molecular dynamics simulations ${ }^{[37]}$ with the Car-Parrinello ${ }^{[38]}$ method have been performed with the CPMD program ${ }^{[39]}$ on a system made up by 27 pyrrole molecules within a cubic box of $14.60 \AA$ with periodic boundary conditions. The dimensions of the simulation box have been chosen in agreement with the experimental density at ambient conditions. ${ }^{[40]}$ The potential energy has been modeled with BLYP exchange ${ }^{[41]}$ and correlation $^{[42]}$ functionals. Norm-conserving Martins-Troullier ${ }^{[43]}$ pseudopotential along with the Kleinman-Bylander ${ }^{[44]}$ decomposition have been adopted to describe the core region of each atom. A plane wave expansion truncated at 60 Ry has been used for the valence electrons. The equations of motion have been integrated for about $38.4 \mathrm{ps}$ in the NVE ensemble using a time step of 4 au ( $\sim 0.096 \mathrm{fs})$ and a fictitious electron mass of 400 au. Van der Waals interactions have been taken into account with the approach proposed by Grimme. ${ }^{[45]}$

\section{$2.4 \mid$ Analysis}

Density $(\rho)$, diffusion coefficient $(D)$, and static dielectric constant $(\varepsilon)$ have been estimated using standard tools provided with GROMACS. In particular, the total dipole moment autocorrelation function was used to calculated $\varepsilon$ according to the following equation:

$$
\epsilon=1+\frac{4 \pi}{3 V k_{B} T}\left(<M^{2}>-<M>^{2}\right)
$$

To calculate the heat of vaporization, $\Delta H_{\text {vap, }}$ a gas-phase simulation of $2 \mathrm{~ns}(\delta t=0.2 \mathrm{fs})$ has been added. ${ }^{[46]}$ Structural analysis and dimers lifetime were performed with the TRAVIS package. ${ }^{[47,48]}$

The relative orientation of first neighbor pyrrole molecules with respect to a reference one was also investigated by means of cluster analysis. In particular, we have used the K-medoids algorithm, ${ }^{[49]}$ a derivative of the popular K-Means (KM) algorithm, which partitions the data points into $\mathrm{N}$ predefined clusters in an iterative manner. Initially each point is assigned to the cluster with nearest centroid based on distance. In each iteration, the cluster centroids are recomputed by averaging the distance between all points in a cluster and the process continues until the cluster assignment 
TABLE 2 Bulk properties

\begin{tabular}{llll} 
Model & $\varepsilon$ & $\rho\left(\mathrm{kg} / \mathrm{m}^{3}\right)$ & $\Delta H_{\text {vap }}(\mathrm{kcal} / \mathrm{mol})$ \\
\hline Exp. $^{[40,52]}$ & 7.92 & 967.0 & 10.78 \\
\hline Ref. $(\mathrm{OPLS} / \mathrm{AA})^{[16]}$ & $4.0 \pm 0.1$ & $985.6 \pm 0.1$ & $10.55 \pm 0.01$ \\
\hline Ref. $(1.27 \mathrm{CM} 5+\mathrm{OPLS} / \mathrm{AA})^{[53]}$ & - & 996.0 & 12.37 \\
\hline Ref. $[17]$ & 8.14 & - & $10.83 \pm 0.01$ \\
\hline This work & $7.8 \pm 0.2$ & $969.0 \pm 0.2$ & $10.5 \pm 0.2$
\end{tabular}

remains unchanged. In $\mathrm{KM}$, a real data point closer to the centroid is assumed as cluster center (medoid) and the data points are instead assigned to the nearest medoids. Clustering was performed using the pyclustering library (v1.49). The optimal number of clusters was determined using the Calinski-Harabaz score. ${ }^{[50]}$ Clustering was performed using an in-house code written in Python and using the Numpy and Scipy libraries. ${ }^{[51]}$

\section{3 | RESULTS}

\section{$3.1 \mid$ Bulk properties}

The proposed model permits to obtain macroscopic properties in remarkable agreement with experiments. In particular, the computed static dielectric constant (7.8) is in agreement with the experimental value (7.92), contrary to the value obtained with the OPLS/AA FF (4.0). Only a polarizable $\mathrm{FF}^{[17]}$ was able to deliver a correct value. The density obtained with our model shows a good agreement with experiments, whereas the heat of vaporization is in line with OPLS/AA and again in agreement with experiment. Furthermore, we also report in Table 2 the values obtained using the 1.27CM5 + OPLS/AA model by Dodda et al. ${ }^{[53]}$ to show that a simple scaling of the partial charges is not sufficient to reproduce the pyrrole bulk properties. Using the Einstein relation, we have also derived the $D$ coefficient obtaining a value of $1.1 \pm 0.1 \times 10^{-5} \mathrm{~cm}^{2} / \mathrm{s}$. Unfortunately no experimental counterpart is available for the pure pyrrole solution.

\subsection{Structural analysis}

The structure of liquid pyrrole has been studied by Gontrani et al. ${ }^{[15]}$ with X-ray diffraction experiments, which allowed to obtain the static structure factor $(S(q))$. A direct comparison with the experimental findings it is not possible, because a sharpening factor has been applied to the experimental curve. However, we computed the static structure factor from MD simulation observing a good agreement in the position of $S(q)$ relative maxima and minima (Figure 2 and Table 3). In particular, the estimated $S(q)$ presents a principal peak at $1.5 \AA^{-1}$ approximately, followed by four less intense peaks (Figure 2A, black continuous line) in agreement with experimental results. In addition, the peak around $9.5 \AA^{-1}$ presents a shoulder at $12 \AA^{-1}$ as in the Exp. $S(q)$. In Figure 2A, the static structure factor calculated considering intramolecular and intermolecular contributions separately, is shown. As could be expected, the peaks at $q$ values larger than $5 \AA^{-1}$ are due to the intramolecular interactions, while $S(q)$ calculated in $q$ range from 0 to $5 \AA^{-1}$ refer to intermolecular ones. To ascribe the intermolecular contribution to specific interactions, we calculated the $S(q)$ considering only (i) the interactions between nitrogen and ring atoms ( $\mathrm{N}$... Ring), (ii) between $\mathrm{C} 3$ and $\mathrm{C} 4$ atoms (C3C4 Atoms). Considering in a first

(A)

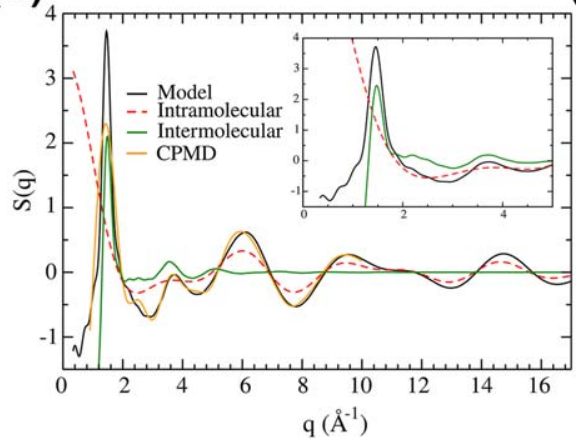

(B)

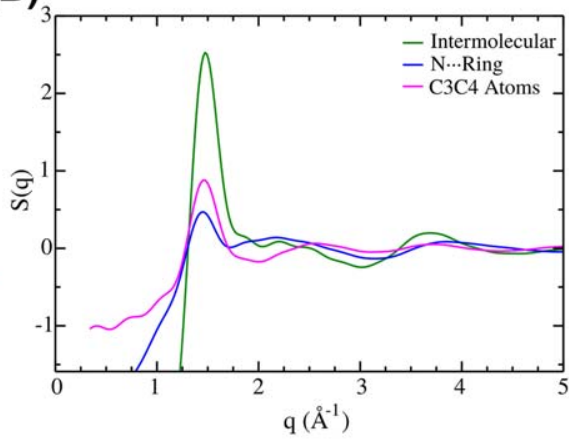

FIGURE 2 A, Theoretical pyrrole static structure factor $S(q)$ derived from MD (black continuous line) and from CPMD (orange continuous line) simulations. In green continuous line the intermolecular contribution and in red dashed line the intramolecular one, both obtained from MD simulation. In the insert low $q$ values only for MD simulation data. B, In green continuous line the intermolecular contribution and its components obtained from MD simulation: in blue line the interactions between nitrogen and ring atoms, and in pink line between $\mathrm{C} 3$ and C4 atoms 
TABLE $3 \quad S(q)$ maxima position

\begin{tabular}{lllllll} 
& \multicolumn{1}{l}{ Peak $\left(\AA^{-1}\right)$} & & \\
Model & 1 & 2 & 3 & 4 & 5 & 5 \\
Exp. $^{\mathrm{a}[15]}$ & 1.4 & 3.6 & 5.9 & 9.5 & 11.2 & 15.5 \\
MD & 1.5 & 3.7 & 6.1 & 9.7 & 11.6 & 14.8 \\
CPMD $^{\mathrm{b}}$ & 1.4 & 3.6 & 5.9 & 9.4 & - \\
\hline
\end{tabular}

${ }^{\mathrm{a}}$ The experimental data have been digitalized, therefore, they could be affected by errors.

bWe have reported only the values compatible with box dimension.

approximation that it is possible to calculate interatomic distances applying the relation $r=2 \pi / q$, we can attribute the N $\cdots$ Ring and the C3C4 Atoms peaks, at around $2 \AA^{-1}$ and $1.5 \AA^{-1}$, respectively, to the T-shaped configurations. Instead the $\mathrm{N} \cdots$ Ring peak at around $1.45 \AA^{-1}$ does not involve rings in the first shell since the intermolecular distances between the nitrogen atom and the pyrrole ring are about $4.35 \AA$. The $S(q)$ has been calculated using also the CPMD (Figure 2) simulation although the region at $q$ values less than $0.5 \AA^{-1}$ are affected by error due to the box dimensions. Result is in line with the classical simulation and comparable with experiment. Using $S(q)$ we can only obtain mutual distance information, rather than angular distribution; for this reason we compared the results of the classical sampling with those issuing from the CPMD simulation. Figure 3 shows a comparison of combined distribution functions between the ring center (RC) ..H1 RDF, a, and the angle distribution $\alpha$ (for definition see ball and stick diagram of Figure 3) found in our simulation with results coming from the CPMD simulations. This confirms that our model describes with good accuracy the intermolecular interactions.

To obtain further details on the liquid structure, we calculated the radial distribution function (RDF) between selected sites. The RDF between the pyrrole center of masses (COM) is shown in Figure 4A; the curve features a local maximum at $4.3 \AA$ and a global one at $5.45 \AA$. The results are in agreement with CPMD simulation. The integral values computed within the corresponding minimum are 1.9 and 13.2 respectively, showing that each molecule has a first solvation shell made up by about two molecules and a second solvation shell by about 11 . Furthermore the first maximum position, $4.3 \AA$, is in good agreement with the experimental value issuing from a microwave spectroscopy study. ${ }^{[14]}$ Additional information about the presence of T-shaped configurations can be gained from the intermolecular RDF between $\mathrm{H} 1$ (for atom labeling see Figure 1) and N, C2, C3 atoms (Figure 4B). The RDFs show a peak at the same position, around $2.45 \AA$, allowing to identify T-shaped aggregations in our simulation, as well as for the CPMD simulation. The integral value for the three curves up to the first minimum is 1 . The H1 . N RDF presents the same shape found in a previous work $^{[53]}$ and the peak at $4.3 \AA$ has also been identified using $S(q)$. The presence of the T-shaped configurations has be confirmed by inspection of the combined distribution function (Figure 5) of the angle $\gamma$, formed by pairs of vectors perpendicular to the aromatic ring (Figure 5 diagrams, green arrows), and the angle $\beta$, formed by pairs of vectors along the $\mathrm{N}-\mathrm{H} 1$ bond (magenta arrows). The analysis has been performed imposing a condition on COM ...COM distance: in one case it ranges from $0 \AA$ to $4.6 \AA$ (Figure $5 A$ ), in another from $4.6 \AA$ to $7.25 \AA$ (Figure $5 B$ ). It is evident that the majority of interacting molecules present a T-shaped configuration; at longer distances parallel conformations are also sampled.
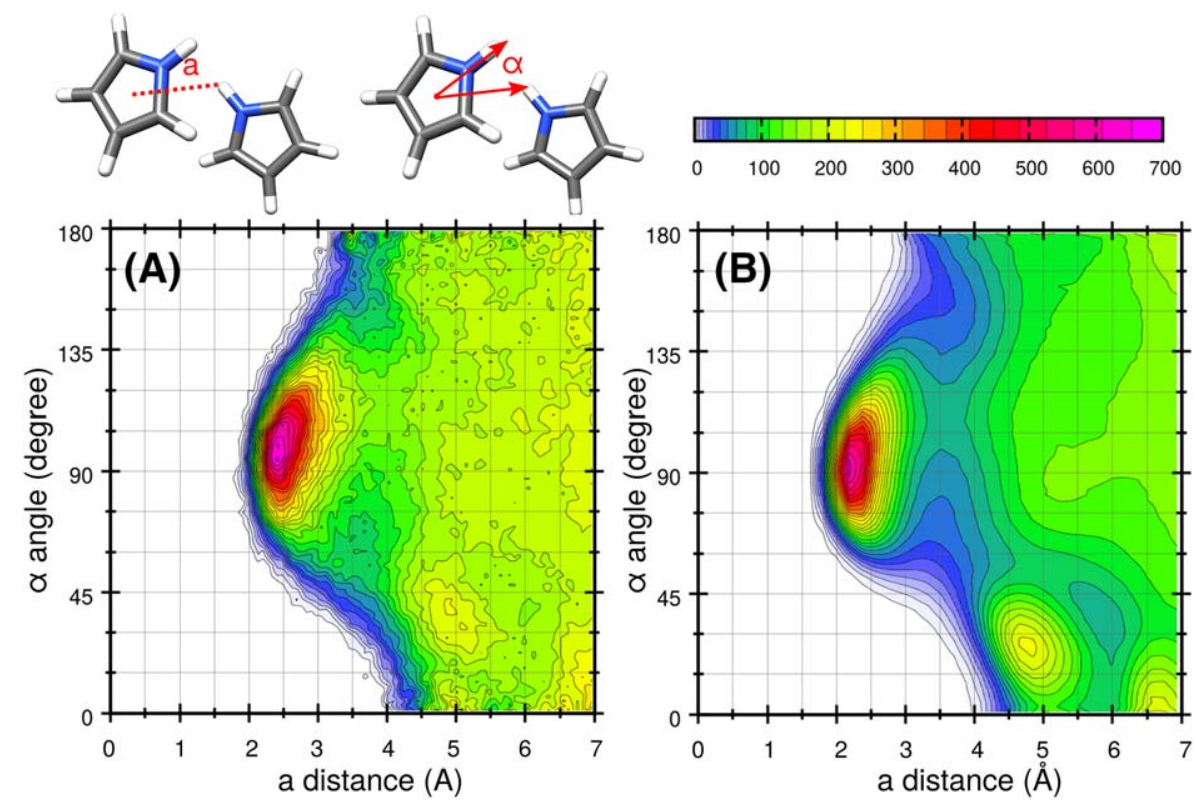

FIGURE 3 Combined distribution function between the a distance and $\alpha$ angle. Results obtained from CPMD (A) and MD (B) simulations 
(A)

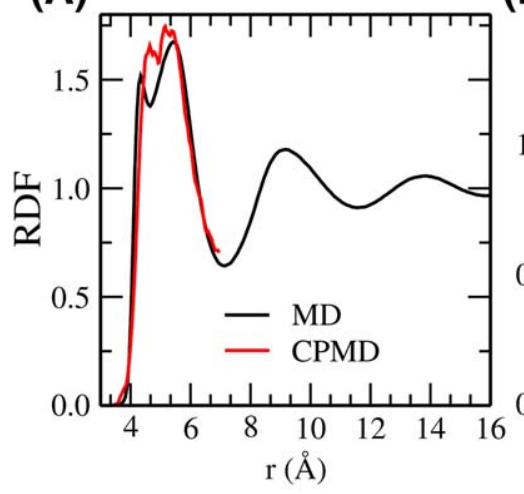

(B)

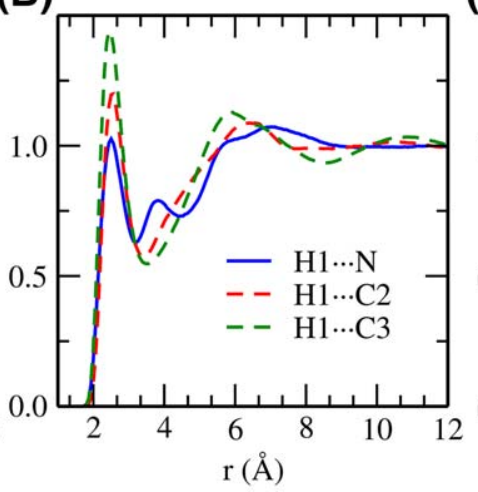

(C)

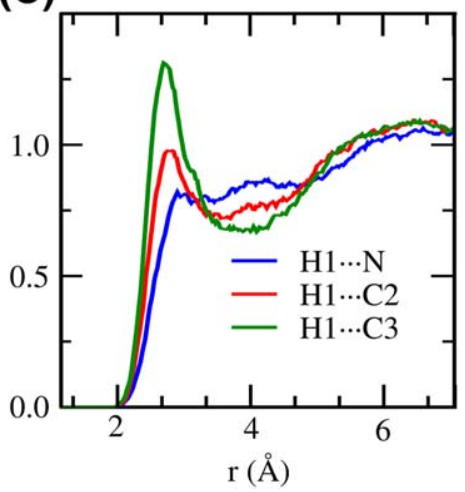

FIGURE 4 A, Radial distribution function between centers of mass (COM). In black continuous line the result obtained from MD simulations, in red continuous line from CPMD. B, Intermolecular radial distribution function obtained from MD simulation between H1 atom and N (blue solid line), C2 (red dashed line) and C3 (green dashed line) atoms. C, Intermolecular radial distribution function between $\mathrm{H} 1$ atom and N (blue solid line), C2 (red dashed line), and C3 (green dashed line) atoms obtained from CPMD

\section{3 | Clusters analysis}

The solvation structure of liquid pyrrole was further investigated by a cluster analysis on pair of pyrrole molecules extracted from the MD trajectory. Using K-medoids, ${ }^{[49]}$ we employed the distances between nitrogen ( $\left.\ldots \mathrm{N}\right), \mathrm{C} 3(\mathrm{C} 3 \ldots \mathrm{C} 3)$, and C4 (C4 ‥C4) atoms with a $4.65 \AA$ cut-off (in other words, the relative orientation of rigid molecular planes was approximated by a triangle). K-medoids was run imposing a number of clusters ( $k$ ) between 2 and 7; validation indicated an optimal clustering at $k_{\text {best }}=3$. The three clusters (see Figure 6) correspond to a T-shaped and two "anti" Tshaped structures; most frames (see Table 4) presented a T-shaped structure with the nitrogen atom of one pyrrole molecule pointing toward the molecular plane of the other, while a in comparable amount of structures either the C3 or C4 carbon atoms were pointing toward the molecular plane; Figure 6 (lower panels) shows the corresponding centroids. It is noteworthy that it is not possible to distinguish clusters by observation of angular distributions and COM.... COM distances (see Figures 4B and 5A).

\section{4 | Multipolar interactions}

To obtain a more detailed interpretation of the intermolecular interactions which occur in liquid pyrrole and to justify the most relevant molecular orientations between neighbor molecules, the contribution due to the dipole-dipole, dipole-quadrupole, and quadrupole-quadrupole interactions
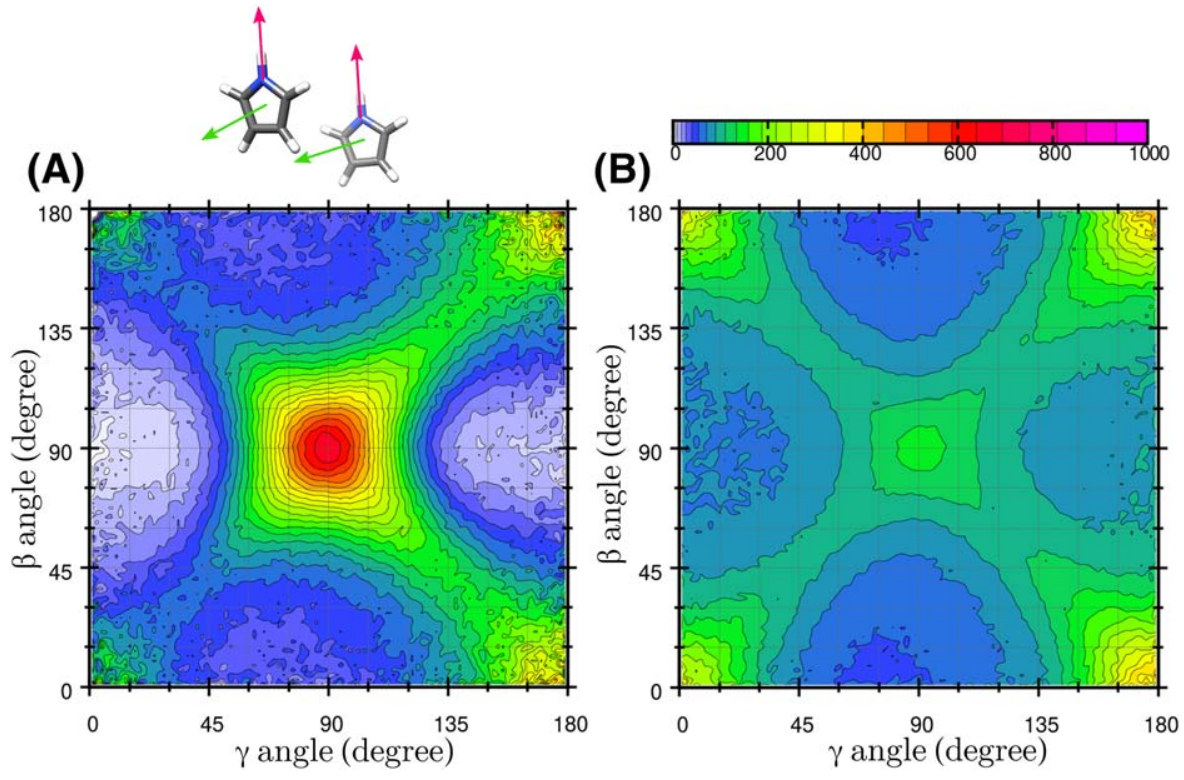

FIGURE 5 Combined distribution function obtained from MD simulation between the angles distribution formed among the vectors perpendicular to pyrrole planes (in green in insert, $\gamma$ ) and the vectors lying on $\mathrm{N}-\mathrm{H} 1$ bond (in magenta in insert, $\beta$ ) calculated imposing a condition on COM ...COM distance, A, from $0 \AA$ to $4.6 \AA$; B, from $4.6 \AA$ to $7.25 \AA$ 

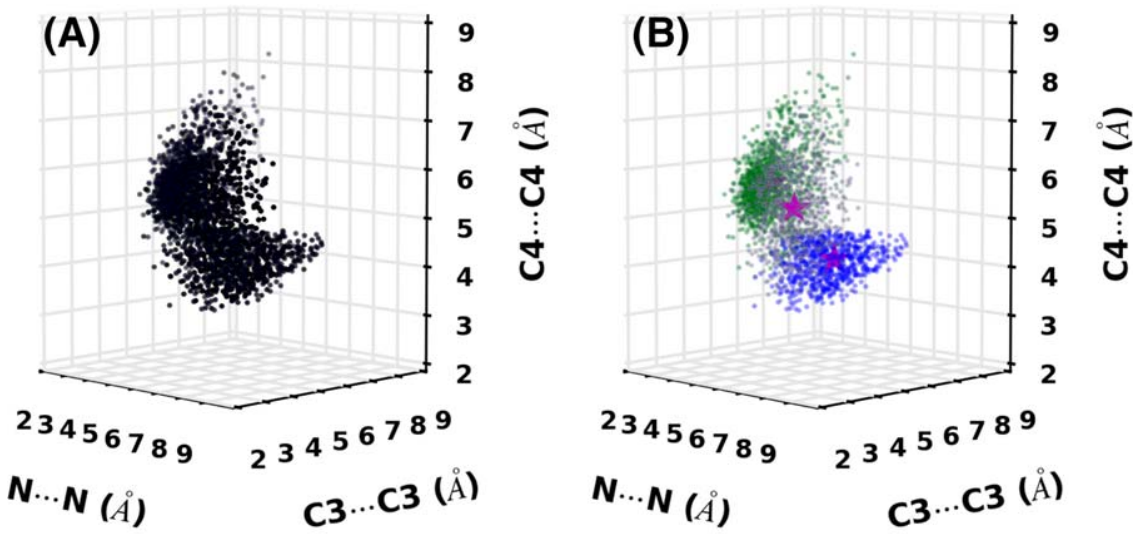

(C)

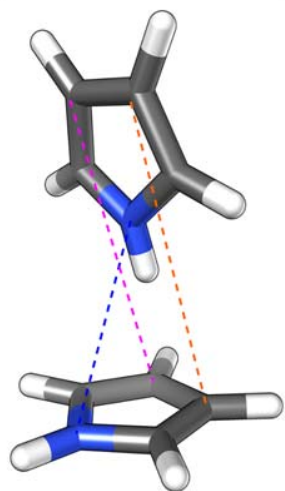

(D)

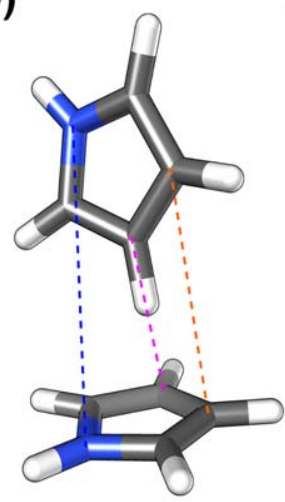

(E)

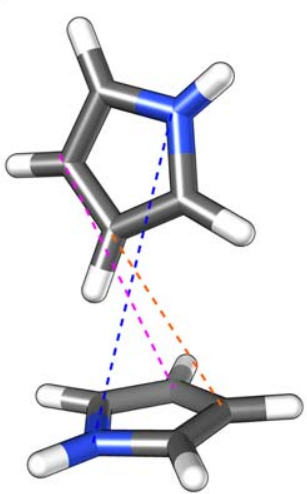

FIGURE 6 K-medoids results from MD simulation. A, Distribution of data points in the selected feature space. B, Separation of data points in clusters; green, gray, and blue (semitransparent) dots indicate clusters; the bigger magenta stars indicate the position of centroids. C-E, Centroid structures of the three obtained clusters; C, Cluster 1, D, Cluster 2, E, Cluster 3

have been examined separately. In fact, it has been hypothesized ${ }^{[24]}$ that the most important contribution in the multipolar expansion in the pyrrole-pyrrole interaction is the quadrupole-quadrupole term, which determines a T-shaped arrangement.

Interaction between two molecules, labeled $A$ and B, has been computed by the multipolar expansion described by Califano et al. ${ }^{[54]}$ In particular, truncating the expansion at the quadrupolar-quadrupolar interaction, the intermolecular potential $V^{A B}$ is:

$$
V^{A B}=-M_{1}^{A} \cdot T_{2}^{A B} \cdot M_{1}^{B}+\frac{1}{3}\left(M_{2}^{A} \cdot T_{3}^{A B} \cdot M_{1}^{B}-M_{1}^{A} \cdot T_{3}^{A B} \cdot M_{2}^{B}\right)+\frac{1}{9} M_{2}^{A} \cdot T_{4}^{A B} \cdot M_{2}^{B}
$$

where $M_{1}$ and $M_{2}$ are the molecular dipole and the traceless quadrupole moments defined as:

$$
M_{1}=\mu_{\alpha}=\sum_{i} q_{i} r_{\alpha}^{i}
$$

and:

$$
M_{2}=\theta_{\alpha \beta}=\frac{1}{2} \sum_{i} q_{i}\left(3 r_{\alpha}^{i} r_{\beta}^{i}-r^{2} \delta_{\alpha \beta}\right)
$$

where $\alpha$ and $\beta$ are the $x, y$, and $z$ Cartesian components, whereas the tensor $T_{S}^{A B}$ is represented by:

$$
T_{S}^{A B}=\nabla^{S} R_{A B}^{-1}
$$

TABLE 4 Centroid intermolecular distances $(\AA)$ and cluster relative size (\% of frames)

$\begin{array}{llll}\text { Cluster/feature } & \text { Cluster 1 } & \text { Cluster 2 } & \text { Cluster 3 } \\ \mathrm{N} \cdots \mathrm{N} & 3.83 & 6.50 & 6.49 \\ \mathrm{C} 3 \ldots \mathrm{C} 3 & 5.58 & 4.20 & 5.68 \\ \mathrm{C} 4 \ldots \mathrm{C} 4 & 5.63 & 5.33 & 4.19 \\ \text { Relative size } & 45.63 & 26.64 & 27.73\end{array}$



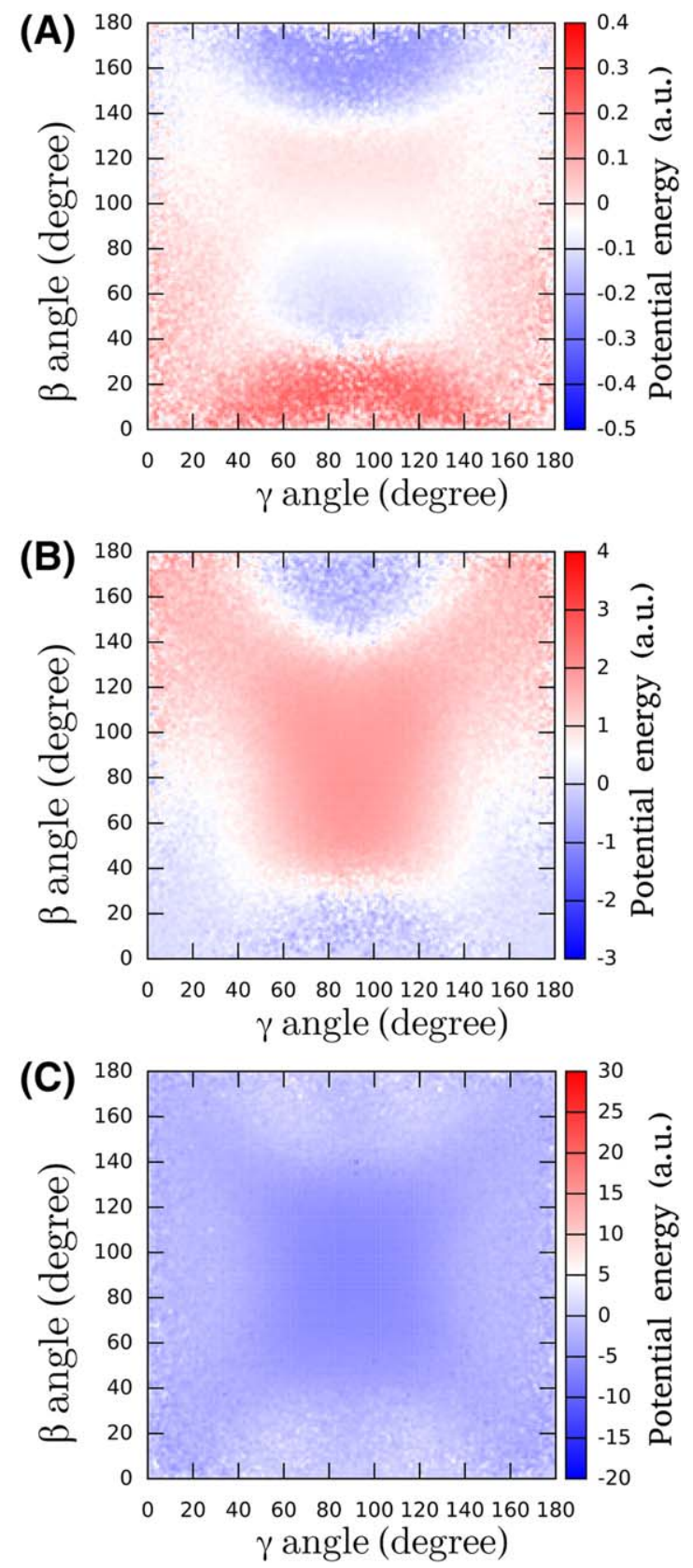

FIGURE 7 Potential energy average values from MD simulation due to the dipole-dipole (A), dipole-quadrupole (B), and quadrupolequadrupole $(C)$ interactions due to neighbor molecules calculated using a cut-off of $4.6 \AA$ for the COM . . COM distance

where $S$ is the rank of the tensor and $R_{A B}$ is the distance between the center of mass of the molecules $A$ and $\mathrm{B}$.

To analyze in detail the interaction in liquid pyrrole, we have adopted the same intermolecular distance cut-off used to obtain the combined distribution functions reported in Figure 5.

In particular, Figure 7 shows separately the average values of the potential energy due to the dipole-dipole, dipole-quadrupole, and quadrupole-quadrupole interactions due to neighbor molecule, using a COM ...COM distance cut-off of $4.6 \AA$. It is apparent that the predominant term arises from the quadrupole-quadrupole interaction, confirming the experimental hypothesis ${ }^{[24]}$ and providing additional information on the molecular arrangement described in the structural analysis.

\section{4 | CONCLUSIONS}

Accurate values of the structural and macroscopic properties of liquid pyrrole have been obtained by performing classical molecular dynamics simulations with a new parameterization of the electrostatic part of the force field. Simple atomic charges have been obtained by performing DFT 
calculations on the pyrrole molecule taking into account the polarization effects due to solvent employing the C-PCM ${ }^{[23]}$ method. The $\mathrm{CM}^{[22]}$ method has been adopted to estimate the atomic charges, which have been adjusted to reproduce multipolar moment components through a fitting procedure. Structural properties from classical molecular dynamics simulation shows a very good agreement with available experimental data ${ }^{[15]}$ and with Car-Parrinello molecular dynamics simulation. The occurrence of the most representative molecular arrangement in the liquid has been justified by analyzing the intermolecular electrostatic interactions in terms of a multipolar expansion. The computed macroscopic properties, that is, dielectric constant, density and heat of vaporization, are in excellent agreement with experiments. It is worth to highlight that the charge adjustment procedure turned out to be of critical importance to reproduce the structural and the bulk properties in agreement with experimental finding. We have shown in past contributions that the use of CM5 method with a proper adjustment of the atomic charges allows to obtain the same accuracy at the molecular and bulk level using standard functional forms for force fields in the case of simple amides ${ }^{[29]}$ and pyridine ${ }^{[30,31]}$ even in different environmental conditions. The approach to determine the atomic charges is general, but the adjustment procedure can be slight modified to improve the directional character of the interactions, opportunely introducing lone pairs (virtual sites) ${ }^{[29,31]}$ or reproducing high order multipoles, ${ }^{[30]}$ as in the present study. It is also important to observe that simpler adjustment procedures, for example, based on the application of a scaling factor ${ }^{[53]}$ may indeed improve overall results when carrying out parameterization of a large number of compounds but fail when a higher degree of accuracy is sought.

\section{ACKNOWLEDGMENTS}

The authors thank SMART Lab technical staff for managing the computing facilities at SNS. Funding from the European Research Council under the European Union's Seventh Framework Programme (FP/2007-2013)/ERC Grant Agreement No. [320951] and the support of Scuola Normale Superiore grant "Giovani Ricercatori 2015" are gratefully acknowledged.

\section{ORCID}

Marina Macchiagodena (iD http://orcid.org/0000-0002-3151-718X

Giordano Mancini (iD) http://orcid.org/0000-0002-1327-7303

Marco Pagliai (D) http://orcid.org/0000-0003-0240-161X

Gianni Cardini (D) http://orcid.org/0000-0002-7292-3555

Vincenzo Barone (iD http://orcid.org/0000-0001-6420-4107

\section{REFERENCES}

[1] W. Arnold, C. T. Edward Eds., Chemistry of Heterocyclic Compounds, Vol. 25, Part IV, Wiley, New York 1983.

[2] H. Fisher, H. Orth, Die Chemie des Pyrrols, Akademische Verlagsgesellshaft, Leipzig 1934.

[3] W. W. Wilkerson, R. A. Copeland, M. Covington, J. M. Trzaskos, J. Med. Chem. 1995, 38(20), 3895.

[4] R. P. Wurz, A. B. Charette, Org. Lett. 2005, 7(12), 2313.

[5] H. Lee, J. Lee, S. Lee, Y. Shin, W. Jung, J.-H. Kim, K. Park, K. Kim, H. S. Cho, S. Ro, S. Lee, S. Jeong, T. Choi, H.-H. Chung, J. S. Koh, Bioorg. Med. Chem. Lett. 2001, 11(23), 3069.

[6] A. G. MacDiarmid, Angew. Chem. Int. Ed. 2001, 40(14), 2581.

[7] Z. Wang, M. Liang, Y. Tan, L. Ouyang, Z. Sun, S. Xue, J. Mater. Chem. A 2015, 3, 4865.

[8] K. Wang, M. Azouz, M. Babics, F. Cruciani, T. Marszalek, Q. Saleem, W. Pisula, P. M. Beaujuge, Chem. Mater. 2016, $28(15)$, 5415.

[9] L. Lubich, S. Oss, J. Chem. Phys. 1997, 106(13), 5379.

[10] S. Millefiori, A. Alparone, J. Chem. Soc. Faraday Trans. 1998, 94, 25.

[11] R. Goddard, O. Heinemann, C. Krüger, Acta Crystallogr. Sect. C 1997, 53(12), 1846.

[12] A. Gmez-Zavaglia, R. Fausto, J. Phys. Chem. A 2004, 108(34), 6953.

[13] W. Gao, H. Feng, X. Xuan, L. Chen, Mol. Phys. 2012, 110(18), 2151.

[14] G. Columberg, A. Bauder, J. Chem. Phys. 1997, 106(2), 504.

[15] L. Gontrani, F. Ramondo, R. Caminiti, Chem. Phys. Lett. 2006, 417(13), 200.

[16] C. Caleman, P. J. van Maaren, M. Hong, J. S. Hub, L. T. Costa, D. van der Spoel, J. Chem. Theory Comput. 2012, 8(1), 61.

[17] P. E. M. Lopes, G. Lamoureux, A. D. Mackerell, J. Comput. Chem. 2009, 30(12), 1821

[18] W. L. Jorgensen, D. S. Maxwell, J. Tirado-Rives, J. Am. Chem. Soc. 1996, 118(45), 11225.

[19] W. L. Jorgensen, J. Tirado-Rives, Proc. Natl. Acad. Sci. USA 2005, 102(19), 6665.

[20] D. van der Spoel et al. Convergence of md simulations of liquids in different software packages, In Preparation, 2017.

[21] C. I. Bayly, P. Cieplak, W. Cornell, P. A. Kollman, J. Phys. Chem. 1993, 97(40), 10269.

[22] A. V. Marenich, S. V. Jerome, C. J. Cramer, D. G. Truhlar, J. Chem. Theory Comput, 2012, 8(2), 527 
[23] V. Barone, M. Cossi, J. Phys. Chem. A 1998, 102(11), 1995.

[24] J. H. Williams, Acc. Chem. Res. 1993, 26(11), 593.

[25] P. W. Fowler, A. D. Buckingham, Chem. Phys. Lett. 1991, 176(1), 11.

[26] C. M. Baker, G. H. Grant, J. Chem. Theory Comput. 2007, 3(2), 530.

[27] C. A. Hunter, J. K. M. Sanders, J. Am. Chem. Soc. 1990, 112(14), 5525.

[28] M. J. Frisch, G. W. Trucks, H. B. Schlegel, G. E. Scuseria, M. A. Robb, J. R. Cheeseman, G. Scalmani, V. Barone, B. Mennucci, G. A. Petersson, H. Nakatsuji, M. Caricato, X. Li, H. P. Hratchian, A. F. Izmaylov, J. Bloino, B. G. Janesko, F. Lipparini, G. Zheng, J. L. Sonnenberg, W. Liang, M. Hada, M. Ehara, K. Toyota, R. Fukuda, J. Hasegawa, M. Ishida, T. Nakajima, Y. Honda, O. Kitao, H. Nakai, T. Vreven, J.A. Montgomery, Jr, J. E. Peralta, F. Ogliaro, M. Bearpark, J. J. Heyd, E. Brothers, K. N. Kudin, V. N. Staroverov, T. Keith, R. Kobayashi, J. Normand, K. Raghavachari, A. Rendell, J. C. Burant, S. S. Iyengar, J. Tomasi, M. Cossi, N. Rega, J. M. Millam, M. Klene, J. E. Knox, J. B. Cross, V. Bakken, C. Adamo, J. Jaramillo, R. Gomperts, R. E. Stratmann, O. Yazyev, A. J. Austin, R. Cammi, C. Pomelli, J. W. Ochterski, R. L. Martin, K. Morokuma, V. G. Zakrzewski, G. A. Voth, P. Salvador, J. J. Dannenberg, S. Dapprich, P. V. Parandekar, N. J. Mayhall, A. D. Daniels, O. Farkas, J. B. Foresman, J. V. Ortiz, J. Cioslowski, D. J. Fox, Gaussian 09 Development Version and Revision i.04p, Gaussian Inc., Wallingford, CT 2010.

[29] M. Macchiagodena, G. Mancini, M. Pagliai, V. Barone, Phys. Chem. Chem. Phys. 2016, 18, 25342.

[30] M. Macchiagodena, G. Mancini, M. Pagliai, G. Del Frate, V. Barone, Chem. Phys. Lett. 2017, 677, 120.

[31] M. Pagliai, G. Mancini, I. Carnimeo, N. De Mitri, V. Barone, J. Comput. Chem. 2017, 38, 319.

[32] S. Pronk, S. Páll, R. Schulz, P. Larsson, P. Bjelkmar, R. Apostolov, M. R. Shirts, J. C. Smith, P. M. Kasson, D. van der Spoel, B. Hess, E. Lindahl, Bioinformatics, 2013, 29(7), 845.

[33] G. Bussi, D. Donadio, M. Parrinello, J. Chem. Phys. 2007, 126(1), 014101.

[34] M. Parrinello, A. Rahman, J. Chem. Phys. 1982, 76(5), 2662.

[35] B. Hess, H. Bekker, H. J. C. Berendsen, J. G. E. M. Fraaije, J. Comput. Chem. 1997, 18(12), 1463.

[36] T. Darden, D. York, L. Pedersen, J. Chem. Phys. 1993, 98(12), 10089.

[37] D. Marx, J. Hutter, Ab Initio Molecular Dynamics: Basic Theory and Advanced Methods, Cambridge University Press, Cambridge, England 2009.

[38] R. Car, M. Parrinello, Phys. Rev. Lett. 1985, 55, 2471.

[39] CPMD. Copyright IBM Corp 1990-2008, Copyright MPI für Festkörperforschung Stuttgart 1997-2001.

[40] D. R. Lide, CRC Handbook of Chemistry and Physics, 90th ed., CRC Press, Cleveland, OH 2009.

[41] A. D. Becke, Phys. Rev. A 1988, 38, 3098.

[42] C. Lee, W. Yang, R. G. Parr, Phys. Rev. B 1988, 37, 785.

[43] N. Troullier, J. L. Martins, Phys. Rev. B 1991, 43, 1993.

[44] L. Kleinman, D. M. Bylander, Phys. Rev. Lett. 1982, 48, 1425.

[45] S. Grimme, J. Comput. Chem. 2006, 27, 1787.

[46] C. H. Bennett, J. Comput. Phys. 1976, 22(2), 245.

[47] M. Brehm, B. Kirchner, J. Chem. Inf. Model. 2011, 51(8), 2007.

[48] O. Hollczki, M. Macchiagodena, H. Weber, M. Thomas, M. Brehm, A. Stark, O. Russina, A. Triolo, B. Kirchner, Chemphyschem $2015,16(15), 3325$.

[49] L. Kaufman, P. J. Rousseeuw, Clustering by means of Medoids. In Statistical Data Analysis Based on the L1Norm and Related Methods, Y. Dodge, North-Holland 1987.

[50] T. Caliski, J Harabasz, Commun. Stat. 1974, 3(1), 1.

[51] E. Jones, E. Oliphant, P. Peterson, et al. SciPy: Open Source Scientific Tools for Python, 2001. http://www.scipy.org

[52] Y. Marcus, The Properties of Solvents, Wiley, Hoboken, New Jersey 1998.

[53] L. S. Dodda, J. Z. Vilseck, K. J. Cutrona, W. L. Jorgensen, J. Chem. Theory Comput. 2015, 11(9), 4273.

[54] S. Califano, V. Schettino, N. Neto, Lattice Dynamics of Molecular Crystals, Springer-Verlag, Berlin Heidelberg, New York, 1981.

How to cite this article: Macchiagodena M, Mancini G, Pagliai M, Cardini G, Barone V. New atomistic model of pyrrole with improved liquid state properties and structure. Int J Quantum Chem. 2018;118:e25554. https://doi.org/10.1002/qua.25554 